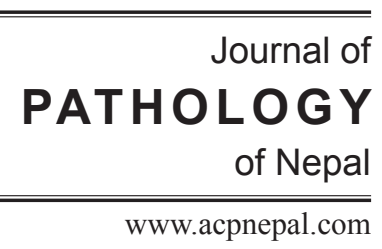

\author{
Case Report
}

\title{
Lymph node infarction and lymphoma
}

\author{
Pai R ${ }^{1}$, Ghartimagar $\mathrm{D}^{1}$, Khadilkar $\mathrm{U}^{1}$, Pai $\mathrm{M}^{1}$, Kini $\mathrm{A}^{2}$ \\ ${ }^{1}$ Department of Pathology, Kasturba Medical College, Mangalore, India \\ ${ }^{2}$ Department of Surgery, Kasturba Medical College, Mangalore, India
}

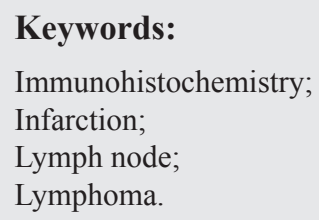

Keywords:

Immunohistochemistry; Infarction; Lymph node; Lymphoma.

\begin{abstract}
Non Hodgkin lymphoma with infarction at initial presentation is rare and can be confused with an acute inflammatory process. A 47 year-old-man presented with complaint of swelling in the left parotid region for 2 weeks which increased in size with severe, continuous and throbbing pain in the last 2-3 days. A clinical diagnosis of parotid abscess was made. Incision and drainage did not yield any pus. Fine needle aspiration cytology showed a highly cellular tumor comprising of somewhat uniform round cells with granular nuclear chromatin. Ghost outline of cytoplasm was noted. A diagnosis of infarcted neoplasm of the parotid gland, probably acinic cell carcinoma was suggested. Histopathologically, it was reported as non-Hodgkin lymphoma with infarction which was confirmed by immunohistochemistry.
\end{abstract}

\section{INTRODUCTION}

Lymph node infarction is associated with concurrent or subsequent malignant lymphoma. ${ }^{1}$ It may also occur following fine needle aspiration biopsy. Fine needle aspiration (FNA) has become an initial diagnostic modality in the evaluation of both non-neoplastic and neoplastic processes involving the lymph nodes. ${ }^{2}$ Here, we report a case of non-Hodgkin lymphoma (NHL) with infarction of a large deep seated lymph node in the parotid region clinically masquerading as an abscess. The potential problems in cytological interpretation of such a case along with role of histopathology and immunohistochemistry are discussed.

\section{CASE REPORT}

A 47 year-old-male presented with history of pain and swelling in the left parotid region for 2 weeks. The swelling increased in size in the last 2-3 days and extended towards

\section{Correspondence:}

Dr. Dilasma Ghartimagar, $M D$

Assistant Professor, Department of Pathology Manipal Teaching Hospital, Pokhara, Nepal.

E-mail: dilasmagm@hotmail.com the lower part of the cheek and neck. Enlargement was associated with severe continuous throbbing pain. He also had fever for 2-3 days. Patient was a known case of hypertension and asthma. There was no history of diabetes mellitus. On examination, a mass about $8 \times 5 \mathrm{~cm}$ in size was seen in the left parotid region extending into the neck with erythematous skin and local rise of temperature. Bilateral submandibular lymph nodes were palpable. Laboratory investigations showed $\mathrm{HIV}$ and $\mathrm{HbsAg}$ status negative. Hemoglobin was $14.9 \mathrm{gm} / \mathrm{dl}$ with total leukocyte count of $7,790 / \mathrm{cmm}$. The peripheral blood smear was normal with a differential count of neutrophils: $64 \%$, lymphocytes: $35 \%$ and eosinophils: $01 \%$ and platelets within normal limits. Liver function test and coagulation profile were also within normal limits. CT scan showed a deep seated well defined hypoechoic lesion in relation to the left parotid gland measuring $4.6 \times 4.4 \times 2.6 \mathrm{~cm}$ in size. A clinical diagnosis of parotid abscess was made and antibiotic treatment was started. Incision and drainage did not yield any pus. Since no clinical improvement was seen FNA cytology was done. Cytology smears were highly cellular and made up of large sheets and groups of round cells with dense 


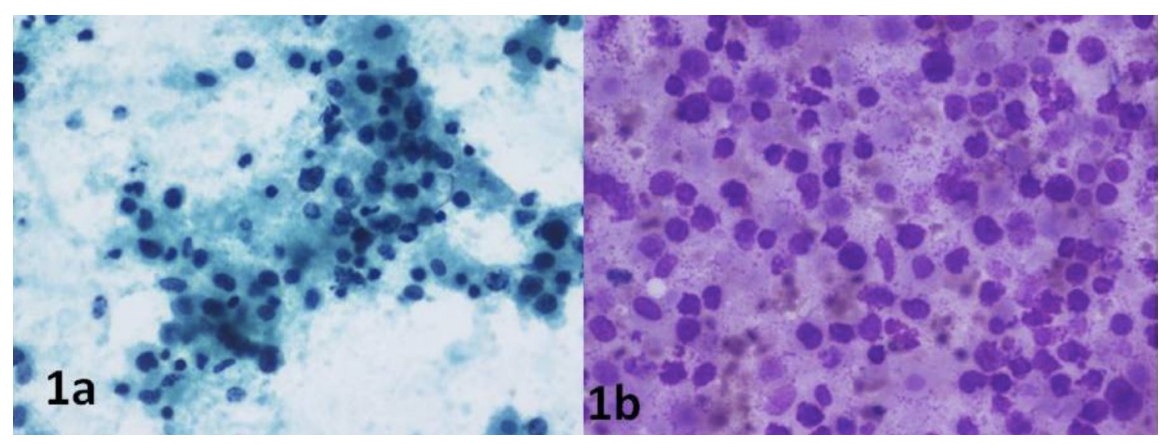

Figure 1: a.Tumor cells with dark nuclei and necrosis in the background (Pap Stain, X400), b. Cellular smear with ghost outline of cytoplasm (Leishman stain X 400).

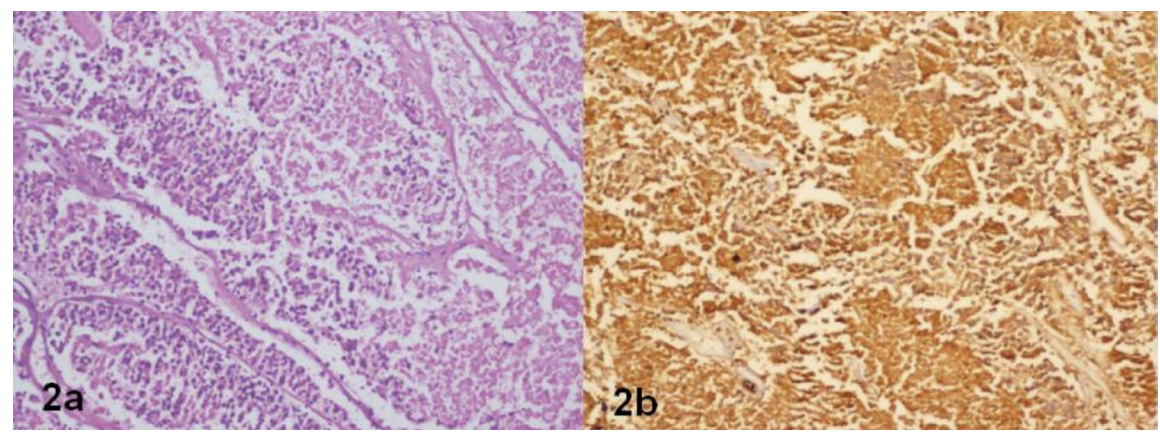

Figure 2: a. Histopathology showing mainly infarcted tumor with few viable tumor cells (H\&E stain, X100),

b. Tumor cells showing diffuse and strong positivity with CD20.

granular cytoplasm and dark mildly pleomorphic nuclei in Pap stained smears (fig.1a). In air dried Leishman stained smears, the ghost outline of cytoplasm was seen and nuclei appeared finely granular. The nuclear details could not be appreciated (fig.1b). The background showed granular necrotic material, bare nuclei and few macrophages. A diagnosis of infarcted neoplasm of the parotid gland, probably acinic cell carcinoma was suggested. Surgery was done which revealed a mass of $5 \times 4 \times 2 \mathrm{~cm}$ size located below the sternocleidomastoid muscle. The excised mass was well encapsulated and cut surface was lobulated, solid, homogenous with areas of necrosis. Histopathologically, the mass represented a lymph node almost entirely replaced by an infarcted tumor (fig.2a). The peripheral viable areas showed monotonous population of lymphoid cells. Focally subcapsular sinus and a reactive lymphoid follicle were seen. A diagnosis of non-Hodgkin lymphoma with infarction was made. Immunohistochemically, the tumor cells were diffusely and strongly positive for CD45 and CD20 (fig.2b) and negative for CD3. A diagnosis of non-Hodgkin lymphoma diffuse, large B cell type was confirmed.

\section{DISCUSSION}

Lymph node infarction refers to a syndrome of spontaneous coagulative necrosis of lymph node that is frequently associated with concurrent or subsequent malignant lymphomas. Lymph node infarction may also follow FNA. The incidence of malignant lymphoma in patients with lymph node infarction varies from as high as $89 \%$ to as low as $32 \%$ in published series. ${ }^{3}$ FNA is recognized as an effective diagnostic tool in the pre-operative diagnosis and staging of various tumors throughout the body. ${ }^{2}$ Initial reports of spontaneous lymph node infarction suggested vasculitis, vascular thrombosis or mechanical vascular obstruction as possible etiologies. The majority of the lymph nodes with infarction were located in the head and neck region.

The differential diagnosis of lymph node infarction includes necrotizing lymphadenitis (Kikuchi's lymphadenitis), the mucocutaneous lymph node syndrome (Kawasaki's syndrome) and necrotic malignant tumors. The necrosis of Kikuchi's lymphadenitis is usually focal, with nuclear karyorrhexis, fibrin deposits and collections of large mononuclear cells. Mucocutaneous lymph node syndrome may show fibrin thrombi in smaller vessels accompanied by patchy infarcts. Metastatic tumors to lymph node, particularly melanoma can undergo spontaneous necrosis in the absence of prior therapy.

Therefore, thorough examination of the lymph node is mandatory and multiple blocks of tissues must be taken for proper diagnosis. Immunohistochemistry is extremely useful to determine the lineage of the necrotic tumor. ${ }^{6}$ In 
our case, CD45 positivity was diffuse and strong in all the necrotic as well as viable malignant cells indicating a lymphomatous process. The tumor cells were CD20 positive and CD3 negative confirming the diagnosis of NHL, diffuse large B cell type.

Lymph node infarction is a distinctly uncommon condition and is seen in two different sets of situations. One, infarction of non-neoplastic nodes due to interference with blood or lymph flow. The other is the infarction of the lymphoma which may be spontaneous or following FNA. ${ }^{7}$ In a study of 51 cases of lymph node infarction over a period of 30 years, fourteen cases of malignant lymphoma was found synchronously with the infarct. Remaining 37 cases were with apparently 'benign' lymph node infarction, out of which 6 showed manifestation of malignant lymphoma in the follow-up period. ${ }^{8}$

A case of NHL with infarction which presented as a lump in the submandibular region of one month duration was clinically diagnosed as sialadenitis with suspected sialolithiasis is documented.7 Our case clinically presented as an abscess and the infarction in the lymphomatous node was spontaneous.

\section{CONCLUSION}

It is important for the pathologists to be aware of lymphomas presenting initially with pain and swelling due to infarction mimicking clinically an acute inflammatory process. On FNA, the infarcted lymphoma cells may be difficult to distinguish from other neoplasms. Lymph node biopsy and application of immunohistochemistry is mandatory for a definitive diagnosis. The immuno markers retain their antigenicity even following infarction. Hence, it is important to remember that markers can be applied on the infarcted lymphomas.

\section{REFERENCES}

1. Strauchen JA, Miller LK. Lymph node infarction. An immmunohistochemical study of 11 cases. Archives Pathol and Lab Med 2002;27:60-3.

2. Nasuti JF, Gupta PK, Baloch ZW. Clinical implications and value of immunohistochemical staining in the evaluation of lymph node infarction after fine needle aspiration. Diagn Cytopathol2001;25:104-7. CrossRef

3. Angelucci D, Artese L, De Lutiis M, Rosini S, Pizzicannella G. Primary lymph node infarct and malignant non Hodgkin's lymphoma. Description of a case. Pathologica1989;81:83-9. PMid:2748211

4. Vega F, Lozano MD, Alcalde J, Pardo-Mindan FJ. Utility of immunophenotypic and immunogenotypic analysis in the study of necrotic lymph nodes. Virchows Arch1999;34:245-8. CrossRef

5. Stamos JK, Corydon K, Donaldson J, Shulman ST. Lymphadenitis as the dominant manifestation of Kawasaki disease. Pediatric1994;93:525-8.

6. Chu PG, Chang KL, Arber DA, Weiss LM. Practical application of immunohistochemistry in hematolymphoid neoplasms. Ann Diagn Pathol1999;3:104-33. CrossRef
7. Bargava S, Tiwari CP, Arun A. Lymph node infarction and lymphoma. Indian J Cancer1989;26:233-9.

8. Maurer R, Schmid U, Dacies JD, Mahy NJ, Stansdeld AG, Lukes RJ. Lymph-node infarction and malignant lymphoma: a multicentre survey of European, English and American cases. Histopathology 1986; 10:571-88. CrossRef 